\title{
Diagnóstico del uso de plaguicidas en el cultivo de tomate riñón en el Cantón Paute
}

\author{
José Reinoso \\ Facultad de Ciencias de la Hospitalidad, Universidad de Cuenca, Cuenca, Ecuador. \\ Autor para correspondencia: jose.reinoso@ucuenca.edu.ec \\ Fecha de recepción: 3 de febrero de 2015 - Fecha de aceptación: 17 de junio de 2015
}

\section{RESUMEN}

El cultivo del tomate es muy susceptible al ataque de plagas y enfermedades, por lo que los agricultores en el mundo y el Cantón de Paute -lugar donde se ha realizado la investigación- utilizan plaguicidas para su prevención y control. Los agricultores en esta zona no respetan las recomendaciones de los fabricantes; aplicando los plaguicidas a sus cultivos de forma incontrolada. Por falta de la medición sistemática de los residuos de plaguicidas en los tomates cosechados, el riesgo de intoxicación es muy probable. Adicional, la tecnología para eliminar los residuos de plaguicidas de las superficies de las verduras y frutas, todavía no está disponible. Se organizó un estudio descriptivo transversal entre septiembre del año 2014 y febrero del año 2015, para obtener un diagnóstico del uso de plaguicidas y de la práctica de aplicación por medio de un cuestionario aplicado a 60 productores de tomate de pequeña escala. La encuesta revela que el conocimiento de los agricultores sobre cual plaguicida usar, la concentración y el tiempo de aplicación es limitado. El estudio recomienda que normas legales que regulen todos los aspectos relacionados con el uso de plaguicidas debieran desarrollarse, junto con la organización de talleres de capacitación. Además, se recomienda que el Ministerio de Agricultura Ganadería Acuacultura y Pesca (MAGAP) proporcione la infraestructura para la medición de los residuos de plaguicidas y, ayude a los productores en la introducción de tecnologías de pos cosecha para reducir los riesgos de salud para el consumo de los productos contaminados.

Palabras clave: Plaguicidas, tomate riñón, productores de pequeña escala, riesgo de salud.

\begin{abstract}
Tomato is very susceptible to pests and diseases, for which farmers worldwide and in the Canton Paute -place where the research was conducted- use pesticides for their prevention and control. Typical for the farmers in the study area is that they do not respect the recommendations of the manufacturers; they apply pesticides in a rather uncontrollable manner. By lack of the systematic measurement of pesticide residues in harvested tomatoes, the risk for food poisoning is not unrealistic. Further, the technology to remove pesticide residues from surfaces of vegetables and fruits is not yet available. To assess in the study area the pesticide use and practice of application, a descriptive transversal study between April and June 2013 was organized by means of a questionnaire administered to 60 small-scale tomato growers. The survey reveals that farmer's knowledge of pesticide use -which pesticide to use, its concentration and time of application- is limited. Therefore, the study recommends that legal standards, regulating all aspects related to the use and handling of pesticides, are developed together with the organization of capacitating workshops. In addition, the study recommends that the Ministry of Agriculture, Livestock and Fisheries (MAGAP) provides infrastructure for measuring pesticide residues, and help producers in the introduction of technology for the postharvest treatment to reduce health risks for the consumption of pesticide-contaminated vegetables.
\end{abstract}

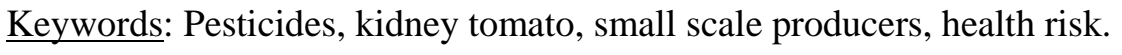




\section{INTRODUCCIÓN}

El tomate riñón (Lycopersicum esculentum Mill) es un cultivo hortícola, herbáceo de ambientes cálidos, con una temperatura en el intervalo de 21 a $23^{\circ} \mathrm{C}$, se lo encuentra cultivado a campo abierto en la costa ecuatoriana; sin embargo, en los valles abrigados de la región sierra, frecuentemente está cultivado bajo invernadero. El proyecto investigativo, los resultados de los cuales se presentan en este artículo, se desarrolló en el Cantón Paute de la provincia del Azuay (Ecuador), específicamente en las parroquias: Bulán, con las comunidades de Padre Urco, Tuntac, Guayan y Suman; El Cabo y Dug Dug, en donde, se organizó un estudio descriptivo transversal entre septiembre del año 2014 y febrero del año 2015, para obtener un diagnóstico del uso de plaguicidas y de la práctica de aplicación por medio de un cuestionario aplicado a 60 productores de tomate bajo invernadero a pequeña escala.

El tomate riñón es muy susceptible al ataque de plagas y enfermedades como el tizón tardío, el tizón temprano y la mosca blanca. El tizón tardío (Phytophthora infestans) es el causante de una de las enfermedades de mayor impacto al tomate y cuando no se controla oportunamente, puede ocasionar la pérdida total del cultivo. En las hojas se observan manchas pardas irregulares que con el tiempo en el envés presentan un fino algodoncillo blanco grisáceo en sus bordes; varias manchas se unen para formar otras más grandes que cubren casi toda la hoja, que luego muere (Rosales \& Romero, 1999). El tizón temprano (Alternaria solani) en el tomate ocasiona tizones en las hojas y que el fruto se pudra. La enfermedad se presenta en cualquier época del desarrollo del cultivo, puede ser causante de pérdidas hasta de $30 \%$ en condiciones favorables para su desarrollo (Rosales \& Romero, 1999). La mosca blanca, se presentó por primera vez en Arizona, Estados Unidos en 1998, un biotipo de Bemisia tabaci denominado biotipo "B" o "Poinsettia", el cual se menciona como originario de Irak o Pakistán, e introducido a Estados Unidos probablemente en un cargamento de frutas o verduras. Esta mosca blanca rápidamente se extendió hacia otras zonas agrícolas como el Valle Imperial de California (García, 2009). Desde Norte América, rapidamente se expandió, encontrándose hoy en día prácticamente en todo el continente americano; siendo las hojas y brotes del tomate los más afectados.

Para contrarrestar la presencia de las plagas y enfermedades, los agricultores recurren al uso de insecticidas y fungicidas. Los plaguicidas son sustancias químicas que se utilizan ampliamente contra las plagas y enfermedades de las plantas, y ha conducido en la agricultura comercial a un aumento de la productividad en la granja (Krol et al., 2000). De acuerdo con la Organización Mundial de la Salud el $20 \%$ del uso de plaguicidas en el mundo se centra en los países en desarrollo y este uso está incrementando (OPS/OMS, 2001). Estudios en materia de plaguicidas son considerados importantes con el fin de disminuir el riesgo de intoxicación y ayudar para mejorar las políticas de salud pública (Ecobichon, 2001). Estudios previos han indicado que el uso inseguro de plaguicidas es común en los países en desarrollo (Konradsen et al., 2003). Los plaguicidas son esenciales en las prácticas agrícolas modernas, pero, debido a su actividad biocida y riesgo potencial para el consumidor, el control de residuos de plaguicidas en los alimentos es fuente de preocupación para la población general (Torres et al., 1996).

Se utiliza una amplia gama de plaguicidas para el control de plagas y control de vectores en las zonas agrícolas afectadas, pero muchos de los agricultores no son informados adecuadamente sobre los riesgos asociados a los productos químicos. Como resultado, los agricultores usan plaguicidas sin el pleno conocimiento de su impacto en la salud humana y el medio ambiente (Ajayi, 2000). En diferentes laboratorios con evidencias epidemiológicas se confirma que ciertos plaguicidas están asociados con carcinogénesis, inmunotoxicidad, neurotoxicidad, deterioro conductual, disfunción reproductiva, alteraciones endocrinas, discapacidades de desarrollo, enfermedades de la piel y las vías respiratorias, enfermedades tales como asma (Solomon et al., 2000). La presencia de sus residuos en frutas y verduras puede ser una vía significativa a la exposición humana (European Council, 1984).

Varios son los plaguicidas que se están utilizando en Paute, el área de estudio donde la investigación ocurrió, dentro de los que señalamos, especialmente por el poder residual que puede quedar en el fruto: Captan: N-triclorometiltio-4-ciclohexeno-1,2-dicarboximida; Bala: Clorpirifos +Cipermetrina; Curzate m8: 1-[(EZ)2-ciano-metoximinoacetil-3-etilures]; Cabrio top: Methyl N-(2\{[1-(4-chlorophenyl)-1H-pyrazol-3-yl]oxymethyl\}phenyl)-N-methoxy carbamate; Curacrón 500: 0-4- 
bromo-2-clorofenil O-etil S-propil fosforotioato; Ridomil: Metalaxil-M Metil(R)-2-[(2,6-dimetilfenil)metoxiacetilamino]-ácido propiónico metil éster.

Captan por ejemplo, es un fungicida ftalimida no sistémico utilizado para controlar las enfermedades de muchas frutas, ornamentales y hortalizas cultivos (Extoxnet, 1996). Existe una fuerte evidencia de que captan causa cáncer en ratones hembra y en ratas macho a dosis altas. Adicionalmente, captan es químicamente similar a otros dos plaguicidas, el folpet y captafol que se ha demostrado que producen cáncer en animales de prueba. Los tumores están asociados con el tracto gastrointestinal y, en menor grado, con los riñones (USDA, 1995; U.S. National Library of Medicine, 1995). Estados Unidos de Norte América, clasifica al captan como B2, un probable carcinógeno humano (Reregistration Elegibility Decision, 1999). Los casos de intoxicación por plaguicidas colectiva en Japón se han reportado ocasionalmente debido al consumo accidental de hortalizas contaminadas con plaguicidas. Dado que los productos agrícolas no pueden ser vendidos si contienen plaguicidas que exceda el límite residual, métodos para eliminar eficazmente residuos de plaguicidas en los cultivos necesitan ser desarrollados (Yamaguchi, 2006).

Por otro lado, se han desarrollado estudios en donde se demuestra que tratamientos especiales a los alimentos, ayudan a disminuir la cantidad residual del plaguicida. Algunos investigadores han encontrado que los niveles residuales de captan y procimidona se redujeron en intervalos antes de la cosecha y/o con aplicaciones culinarias, como lavado, pelado y almacenamiento (Teixeira et al., 2004). Varias soluciones de lavado como solución de cloro, agua ozonizada y ácido fuerte han sido probadas para eliminar correctamente incluso residuos de plaguicidas hidrofóbicos durante el proceso de cultivo comercial (Ong et al., 1996; Zohair, 2001; Wu et al., 2003; Pugliese et al., 2004; Ikeuraa et al., 2011). Otros informes han concluido que la solubilidad en agua de los plaguicidas no juega un papel significativo en su capacidad de reducción en diferentes productos lavándolos (Cabras et al., 1997; Krol et al., 2000; Guardia-Rubio et al., 2007). La razón principal para que no haya costos de salud ocasionados por intoxicaciones por plaguicidas, se debe al hecho de que los funcionarios de salud locales no suelen diagnosticar los síntomas en relación con las exposiciones, y no están adecuadamente capacitados para identificar efectos adversos de los plaguicidas (Ngowi et al., 2001; Ngowi et al., 2002).

En este marco se plantea como objetivos de la investigación: Determinar los plaguicidas de mayor demanda por los agricultores y que tendrían efecto residual nocivo, usados en tomate riñón; conocer las plagas y enfermedades de mayor incidencia que atacan al cultivo de tomate riñón y conocer las frecuencias de aplicación de los plaguicidas en el cultivo de tomate riñón por parte de los agricultores. Para conseguir esta información se trabajó con respuestas a una encuesta dirigida a los agricultores y, se ha realizado el levantamiento de la información in situ, denotándose la predisposición positiva para brindar las respuestas a las preguntas formuladas.

\section{MATERIALES Y METODOS}

El proyecto investigativo se desarrolló en el Cantón Paute, que está ubicado en la sierra ecuatoriana sobre un valle interandino que va desde los 2100 a $2300 \mathrm{msnm}$ con temperaturas de 15 a $25^{\circ} \mathrm{C}$; en el noreste de la Provincia del Azuay, en la latitud sur $2^{\circ} 46^{\prime} 55^{\prime \prime}$ y longitud oeste $78^{\circ} 45^{\prime} 6^{\prime \prime}$. Tiene 8 parroquias, dentro de estas existe una urbana, que tiene el mismo nombre y las rurales San Cristóbal, Bulán, El Cabo, Dug Dug, Chicán, Tomebamba y Guaraynag. Tradicionalmente la agricultura y la ganadería son las principales ocupaciones de sus habitantes abarcando un 62\% (según los datos del censo del 2010). Los suelos que predominan en las subcuencas de las zonas media y baja del río Paute, corresponden a Hidrandepts, derivados de materiales piroclásticos, alofánicos, francos, de coloración negra a rojiza, de gran cantidad de retención de agua, aptos para el cultivo de hortalizas, frutales, pastizales y otros (Valarezo, 2004).

Para el proyecto de investigación se consideró unicamente las parroquias Bulán, El Cabo y Dug Dug del Cantón Paute, por ser las más representativas en la producción de tomate riñón. La región posee un clima subtropical templado, lo cual le permite con una ligera modificación -uso de invernaderos- producir el tomate riñón con gran facilidad. Las unidades productoras de tomate que se 
consideraron para el levantamiento de información, pertenecen a productores de pequeña escala, ya que las superficies que abarcan los invernaderos oscilan desde los $3000 \mathrm{~m}^{2}$, hasta los $10000 \mathrm{~m}^{2}$; muy pocos sobrepasan esta superficie.

Para obtener un diagnóstico del uso de plaguicidas y de la práctica de aplicación, se empleó un cuestionario aplicado a 60 productores de tomate de pequeña escala, para conocer básicamente cuáles son las plagas y enfermedades que atacan a su cultivo, los plaguicidas más utilizados para su control, el lugar en donde los adquieren y sobre todo el manejo de los plaguicidas. Se utilizó igualmente un cuestionario dirigido a los propietarios o administradores de los almacenes agropecuarios del Cantón Paute, para conocer cuáles son los plaguicidas de mayor demanda por los agricultores para ser aplicados en el cultivo de tomate riñón; se realizó la comparación de los datos obtenidos de las dos partes, para determinar las coincidencias entre los productos vendidos en los almacenes agropecuarios frente a los utilizados por los agricultores. Las encuestas fueron realizadas por el autor, quien anotaba sobre su cuestionario las respuestas brindadas por los agricultores, dejándose notar en sus respuestas que todos saben leer y escribir, pero su grado de educación mayoritario llega sólo a la educación primaria. Las preguntas relevantes para cumplir con los objetivos de la investigación y, que se plantearon en formato de encuesta, fueron:

- Nombre del propietario o administrador.

- Ubicación (parroquia).

- ¿Cuáles son las plagas más frecuentes que atacan a su cultivo de tomate?

- ¿Cuáles son las enfermedades más frecuentes que atacan a su cultivo de tomate?

- ¿Cuáles son los plaguicidas que usted compra para aplicar a su cultivo de tomate?

- ¿En dónde compra los plaguicidas?

- ¿Cuántas veces aplica usted plaguicidas durante el ciclo de cultivo?

- ¿Cuál es la última fecha de aplicación de plaguicida, antes de la cosecha?

Al ser la cosecha del tomate riñón cíclica -según vayan ganando peso los frutos y su grado de maduración sea aceptable para la recolección-, los agricultores de la zona en estudio realizan esta práctica cada ocho días o en menor tiempo si el caso lo amerita, pero no más allá de los ocho días. Generalmente, la recolección de los frutos lo hacen por la mañana y, por la tarde realizan la fumigación.

\section{RESULTADOS Y DISCUSIÓN}

La encuesta revela que las plagas y enfermedades que están afectando al cultivo de tomate riñón en la zona de influencia del proyecto son, entre las plagas: mosca blanca, minador del tallo, enrollador de la hoja, biñao y caminador de la hoja, como muestra la Fig. 1. Igualmente, las enfermedades que están atacando al cultivo de tomate en la zona del proyecto son: lancha negra, mancha chocolate, botrytis, la bacteria (al parecer es erwinia, pero los agricultores lo identifican con ese nombre), oidio, lancha amarilla, antracnosis y fusarium; igualmente su incidencia se observa en la Fig. 1.

De los resultados de las encuestas correspondientes a las preguntas 1 y 2 y, representados en la Fig. 1, se observa que la mosca blanca (Fig. 1a), se encuentra presente en la totalidad de los cultivos de tomate bajo invernadero (60), al minador del tallo lo encontramos en 52 invernaderos, al enrollador de la hoja en 13, al biñao en 10 y al caminador de la hoja en 9. En cuanto a las enfermedades, se observa que la lancha negra (Fig. 1b) es la enfermedad de mayor incidencia en los cultivos con una presencia en 46 invernaderos; otra de gran magnitud y que se ha constituido en una enfermedad difícil de controlar es la mancha chocolate, que lo encontramos en 38 invernaderos, botrytis lo encontramos en 27, la bacteria en 25, oidio en 10, lancha amarilla en 8 , antracnosis en 7 y fusarium en 2.

Para el control de las plagas los agricultores utilizan insecticidas, siendo la frecuencia de uso en los invernaderos, según se muestra en la Fig. 2a, la siguiente: tryclán en 42 invernaderos, le sigue en importancia de utilización nakar en 21, bala en 18, acetamiprid en 11, curacrón en 8 , sunfire en 6 , belt en 5, lorsban en 3 y cipermetrina en 1 invernadero. Igualmente para el control de las enfermedades se puede apreciar en la Fig. 2b la frecuencia de uso: ridomil en 20 invernaderos, terraclor en 18, topas en 
16 , cursate en 8 , score en 7 , vitavax en 6 , triziman $\mathrm{D}$ en 3 , daconil en 3 , kasumin en 1 , cobre líquido en 1 y cabrio-top en 1 invernadero.

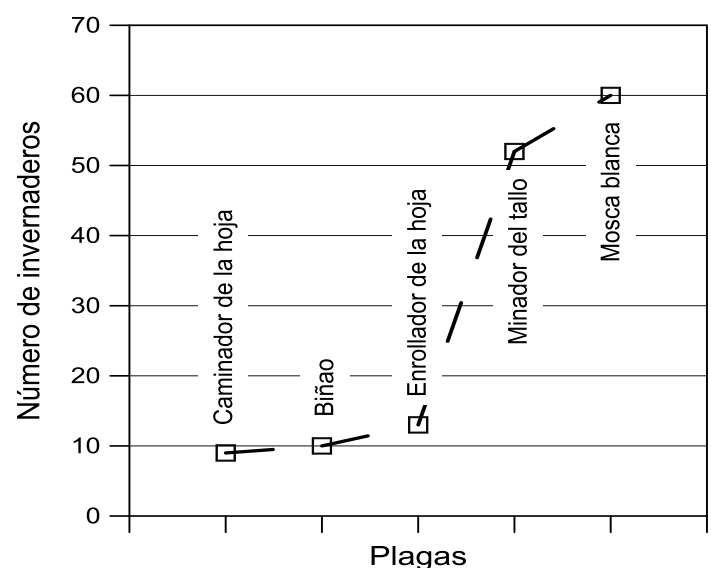

(a)

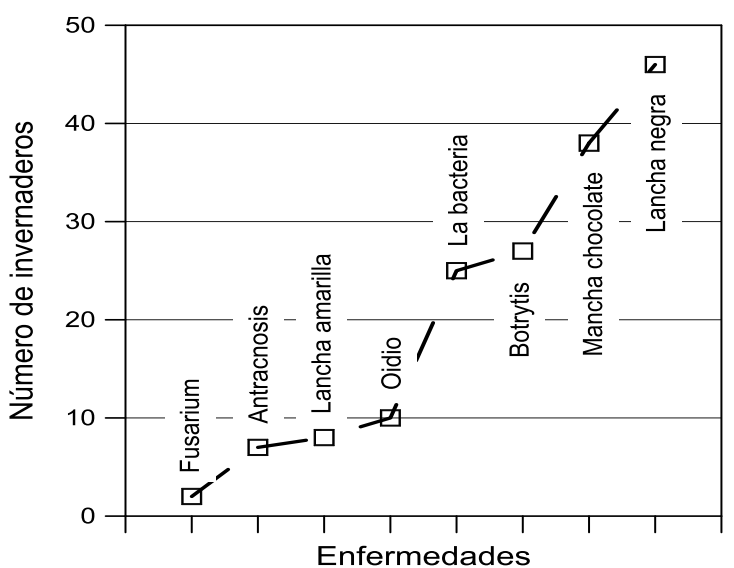

(b)

Figura 1. Número de invernaderos en donde se encuentra plagas (a) y enfermedades (b).

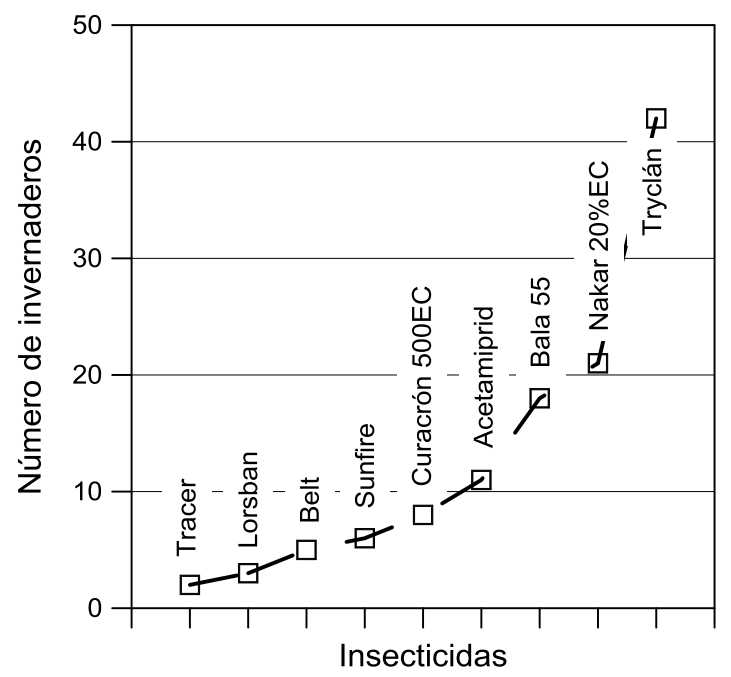

(a)



(b)

Figura 2. Frecuencia de uso de insecticidas (a) y fungicidas (b) por invernadero.

La frecuencia de aplicación de los plaguicidas por parte de los agricultores, para el control de plagas y enfermedades del cultivo de tomate en la zona de influencia del proyeco, así como el tipo de plaguicida utilizado, se han constituido en puntos críticos, ya que la encuesta revela que el conocimiento de los agricultores sobre cual plaguicida usar, así como la concentración y el tiempo de aplicación, es limitado. En la Fig. 3 podemos comparar las frecuencias de aplicación antes de la cosecha recomendadas por el fabricante (Fig. 3b), y las utilizadas por los agricultores (Fig. 3a).

Analizando los resultados estadísticos de la Fig. 3(a) observamos que 35 de los agricultores entrevistados realizan la última aplicación antes de la cosecha a los ocho días; 6 a los nueve días; 10 a los diez días; 1 a los 11 días; 1 a los 12 días; 2 a los 13 días y 1 a los 15 días. El fabricante recomienda que el período de carencia -el lapso de tiempo que tiene que transcurrir entre la última aplicación del plaguicida y la cosecha-, debe ser según el prodúcto, generalmente mayor a los 14 días, salvo el uso de plaguicidas no toxicos, que podrían estar en el rango inferior a los 8 días. En la Fig $3 b$ podemos observar que, para bala se recomienda 21 días como período de carencia, 21 días para curzate, 21 días para cabrio-top, 14 días para curacrón, tryclán, acetamiprid, ridomil y kasumín. Los de baja toxicidad 
están recomendados su uso a los 8 días antes de la cosecha, y entre éstos tenemos: Triziman D, Daconil, Topas, Score, Tracer, sunfire. No se otorga valores a vitavax, terraclor y nakar, por ser fungicidas utilizados como desinfectantes de la semilla, y su uso es antes de la siembra. No se especifica en esta investigación qué plaguicida específicamente está usando el agricultor en su última aplicación, porque hay un desconocimiento generalizado de los productos; algunos agricultores afirman que no conocen lo que están aplicando al cultivo, porque en el almacén, el técnico lo que hace es mezclar unos cuantos productos (plaguicidas) y les entregan indicándoles la cantidad de agua recomendada para que apliquen al cultivo.

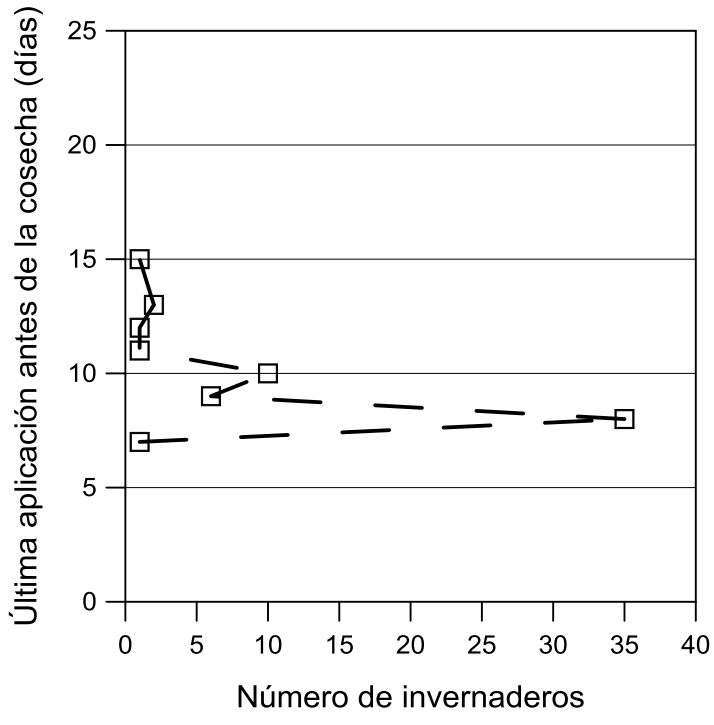

(a)

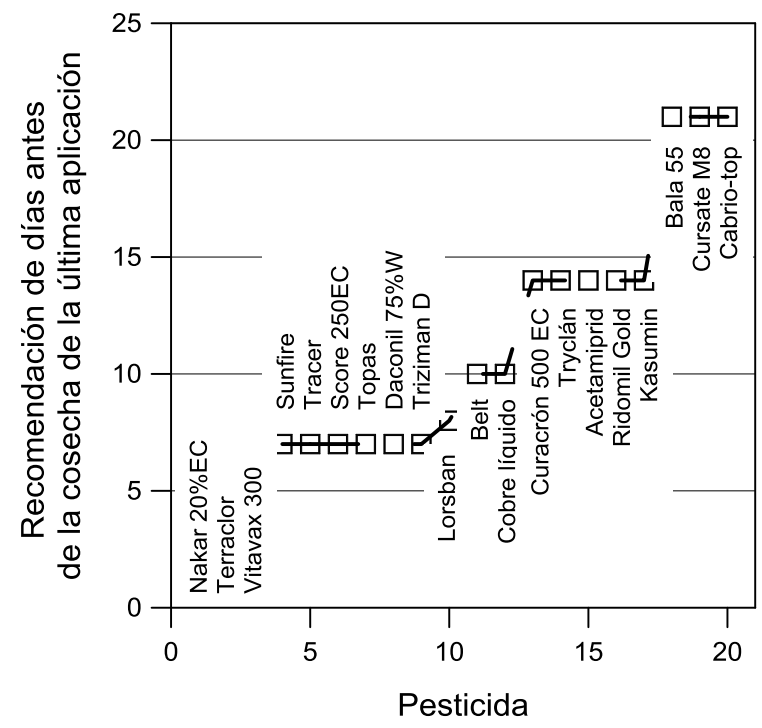

(b)

Figura 3. Frecuencia de los agricultores en función de la última aplicación de plaguicidas en días antes de la cosecha (a) y periodo de carencia recomendado por los fabricantes de plaguicidas (b).

Ante estas circunstancias, el estudio recomienda que debe mejorarse las normas legales que regulan todos los aspectos relacionados con el expendio y uso de plaguicidas; no se desconoce su existencia, pero realmente no se las cumple o no se las quiere cumplir, tanto en expendio de los agroquímicos, así como en la utilización final por parte de los agricultores; resultando el más perjudicado, el consumidor final del producto que lo lleva a su casa, en donde familias enteras pueden ser contaminadas con los plaguicidas. Se debe organizar frecuentemente talleres de capacitación para el manejo de plaguicidas, principalmente a los agricultores y también a los expendedores de agroquímicos, ya que algunos de los propietarios de estos almacenes, no son profesionales calificados; esta es una realidad en todo el Ecuador. Además, se recomienda que el Ministerio de Agricultura Ganadería Acuacultura y Pesca (MAGAP) proporcionen la infraestructura para la medición de los residuos de plaguicidas y, ayuden a los productores en la introducción de tecnologías de postcosecha para reducir los riesgos de salud por el consumo de productos contaminados.

\section{CONCLUSIONES}

Los agricultores, con la finalidad de no perder sus cosechas, están aplicando los plaguicidas al cultivo de tomate, tantas veces ellos consideren necesarias. Hay un interés económico que no les permite refleccionar sobre el daño que puede causar sus cosechas al consumidor final. Si bien, la utilización de algunos plaguicidas está permitido en un período de tiempo de ocho días antes de la cosecha; sin embargo, la mayoría de ellos demanda de mayor cantidad de tiempo entre la última aplicación y la cosecha. Los fabricantes recomiendan en la mayoría de casos, que la última aplicación sea no antes de 
los ocho días, otros lo recomiendan a los catorce días, e inclusive otros a los veintiún días, debido a sus características toxicológicas. Corresponde a las autoridades involucradas en todos los procesos de la producción agrícola en general, el regular estrictamente el manejo de plaguicidas y, que los productos usados sean preferiblemente de procedencia orgánica, biodegradables y no tóxicos.

\section{BIBLIOGRAFÍA}

Ajayi, O.C., 2000. Pesticide use practices, productivity and farmer's health: The case of cotton-rice systems in Cote d'Ivoire, West Africa. Hannover, Germany: A publication of the Pesticide Policy Project, Special Issue Publication Series, No. 3, 172 pp.

Cabras, P., A. Angioni, V.L. Garau, M. Melis, F.M. Pirisi, G.A. Farris, C. Sotgiu, E.V. Minelli, 1997. Persistence and metabolism of folpet in graps and wine. J. Agric. Food Chem., 45(2), 476-479.

Ecobichon, D.J., 2001. Pesticide use in developing countries. Toxicology, 160(1-3), 27-33.

European Council, 1984. Pesticide-advice and recommendations to be used by national and other authorities as well as manufactures concerned with the registration of agricultural and nonagricultural pesticides. $6^{\text {th }}$ ed., Strasburg, Germany, 40 pp.

Extoxnet, 1996. Pesticides information profiles. Extension Toxicology Network. Disponible en http://extoxnet.orst.edu/pips/captan.htm.

Garcia, J.R., 2009. Estudio de evaluación de la efectividad biológica de Movento para el combate de ninfas de mosca blanca (Bemisia sp.) y su fitocompatibilidad en tomate Saladette bajo agricultura protegida. Facultad de Agronomía, Universidad Autónoma de Sinaloa, Sinaloa, México. Disponible en http://www.bayercropscience.com.mx/bayer/cropscience/ bcsmexico.nsf/id/ Abejorros_BCS/\$file/mosca_bca.pdf.

Guardia-Rubio, M. M.J. Ayora-Cañada, A. Ruiz-Medina, 2007. Effect of washing on pesticide residues in olives. J. Food. Sci., 72, 139-143.

Ikeuraa, H., F. Kobayashi, M. Tamaki, 2011. Removal of residual pesticides in vegetables using ozone microbubbles. J. Hazard Mater., 186, 956-959.

Konradsen, F., W. van der Hoek, D.C. Cole, G. Hutchinson, H. Daisley, S. Singh, M. Eddleston, 2003. Reducing acute poisoning in developing countries - options for restricting the availability of pesticides. Toxicology, 192(2-3), 249-261.

Krol, W.J., T.L. Arsenault, H.M. Pylypiw, M.J. Incorvia Mattina, 2000. Reduction of pesticide residue on produce by rinsing. J. Agr. Food Chem., 48, 4666-4670.

Ngowi, A.V., D.N. Maeda, C. Wesseling, T.J. Partanen, M.P. Sanga, G. Mbise, 2001. Pesticidehandling practices in agriculture in Tanzania: Observational data from 27 coffee and cotton farms. Int. J. Occup. Environ. Health, 7, 326-332.

Ngowi, A.V., D.N. Maeda, T.J. Partanen, 2002. Knowledge, attitudes and practices (KAP) among agricultural extension workers concerning the reduction of the adverse impact of pesticides in agricultural areas in Tanzania. Med. Lav., 93, 338-346.

Ong, K.C., J.N. Cash, M.J. Zabik, M. Siddig, A.L. Jones, 1996. Chlorine and ozone washes for pesticide removal from apples and processes apple sauce. Food Chem., 55(2), 153-160.

OPS/OMS, 2001. Plaguicidas y salud. Sección Especial Masica Review. Pesticide Free. Central America Attempts to Revert Indiscriminate Use. San José, Costa Rica.

Pugliese, P., J.C. Moltó, P. Damiani, R. Marín, L. Cossignani, J. Mañes, 2004. Gas chromatographic evaluation of pesticide residue contents in nectarines after non-toxic washing treatments.

J. Chromatogr. A., 1050, 185-191.

Rosales, S.A., N.J. Romero, 1999. Hortalizas plagas y enfermedades. México D.F. (México): Editorial Trillas, 544 pp. 
Solomon, G., O.A. Ogunseitan, J. Kirsch, 2000. Pesticides and human health: A resource for healthcare professionals. Physicians for Social Responsibility and Californians for Pesticide Reform. Disponible en http://www.igc.org/cpr.

Texeira, M.J., A. Aguiar, C.M.M., Alfonso, A. Alves, M.M.S.M. Bastos, 2004. Comparison of pesticides levels in grape skin and in whole grape by a new liquid chromatographic multiresidue methodology. Analytíca Chimica Acta, 513, 333-340.

Torres, C.M., Y. Picó, J. Mañes, 1996. Determination of pesticide residues in fruit and vegetables. J. Chromatogr. A., 754(1-2), 301-331.

USDA, 1995. U.S. Department of Agriculture, National Agricultural Pesticide Impact Assessment Program (NAPIAP). Reregistration Notification Network (RNN), 3(11), 1.1-1.4.

U.S. National Library of Medicine, 1995. Hazardous substances data bank. Bethesda, Maryland, USA.

Reregistration Eligibility Decision (RED), 1999. Office of Pesticide Programs. U.S. Environmental Protection Agency.

Valarezo, C., 2004. Caracterización, distribución, clasificación y capacidad de uso de los suelos en la Región Amazónica Ecuatoriana (RAE). Centro de Estudios y Desarrollo de la Amazonía (CEDAMAZ), Universidad Nacional de Loja, PROMSA, Loja, Ecuador, 201 pp.

Wu, X., G.R. Beecher, J.M. Holden, D.B. Haytowitz, S.E. Gebhardt, R.L. Prior, 2004. Lipophilic and hydrophilic antioxidant capacities of common foods in the United States. J. Agric. Food Chem., 8, 4026-4037.

Yamaguchi, Y., 2006. Environmental and food hygiene approach to pesticide. Seilatsu Eisei, 50(5), 283-290.

Zohair, A., 2001. Behaviour of some organophosphorus and organochlorine pesticides in potatoes during soaking in different solutions. Food Chem. Toxicol., 39(7), 751-755. 